\section{Imaging in Rare Disease: a roadmap for diagnosis}

Giovanni Di Salvo, ${ }^{1}$ Giuseppe Limongelli

'Pediatric Cardiology, Second University of Naples, Monaldi Hospital, Naples; 2Second University of Naples, Monaldi Hospital, Naples, Italy

Declare the past, diagnose the present, foretell the future.

Hippocrates

Rare diseases are a heterogeneous group of conditions including genetic syndromes, inherited metabolic diseases, and neuromuscular disorders with various expression and different organ involvement. A multidisciplinary approach is mandatory for the timely diagnostic recognition and for the proper therapeutic management.

Unfortunately, the diagnosis is generally delayed. However, in the last few years, this field has been characterized by a growing attention due to the availability of innovative therapeutical approaches.

Cardiac involvement is very frequent and it represents the main determinant of patient survival; thus the early identification of cardiac involvement and its etiology is of paramount importance, as some causes require specific treatment and may be correctable, according to the paradigma early diagnosisearly treatment. In this regard non-invasive imaging plays a central role allowing the iden- tification of specific early features helping to make the appropriate diagnosis, and enabling the physician to start the best management strategy. Standard echocardiography is the first-line investigation for detecting a cardiac involvement in systemic disease, but unfortunately, traditional echocardiographic methods often fail to distinguish among these various forms of heart muscle disorders (i.e. differential diagnosis of left ventricular hypertrophy) despite their dissimilarities.

Myocardial deformation imaging, a relatively new approach to asses both global and regional myocardial function, has the potential to become a unique tool for the assessment of such patients as it effectively helps in distinguishing different types of hypertrophy. This non-invasive, ultrasonic based modality may help physicians in the diagnostic evaluation allowing a better allocation of resources and minimizing invasive diagnostic strategies. Cardiovascular magnetic resonance with a single evaluation provides both detailed cardiac and extracardiac structure examination, as well as cardiac tissue characterization.

In this issue of Cardiogenetics, we aimed to describe the potential benefit of the new ultrasonic based techniques and of cardiovascular magnetic resonance in the context of a multidisciplinary diagnosis and management of the principal rare cardiomyopathies. ${ }^{1-3}$

To this purpose, we invited a group of specialists for each disease and imaging modality to describe their day-by-day clinical experience with rare cardiomyopathies, highlighting the advantages but also the limitations of these techniques.
Correspondence: Giovanni Di Salvo, Pediatric Cardiology, Second University of Naples, Monaldi Hospital, Naples, Italy.

E-mail: giodisal@yahoo.it

Received for publication: 13 December 2013. Accepted for publication: 13 December 2013.

This work is licensed under a Creative Commons Attribution NonCommercial 3.0 License (CC BYNC 3.0).

(OCopyright G. Di Salvo and G. Limongelli, 2013 Licensee PAGEPress, Italy

Cardiogenetics 2013; 3:e10

doi:10.4081/cardiogenetics.2013.e10

\section{References}

1. Niemann M, Weidemann F. Echocardiography in Fabry disease. Cardiogenetics 2013;3:e3.

2. Fadel B, Baldini L, Pergola V, et al. Myocardial deformation imaging and rare cardiomyopathies with hypertrophic phenotype: a review focused on Fabry disease, Friedreich ataxia and amyloidosis. Cardiogenetics 2013;3:e4.

3. Palladino A, Nigro G, Politano L. Muscular dystrophies: key elements for everyday diagnosis and management. Cardiogenetics 2013;3:e9. 